\title{
Are "Gap-Fillers" Missing Premisses?
}

\author{
WAYNE GRENNAN Saint Mary's University
}

\begin{abstract}
Identifying the missing or unstated premisses of arguments is important, because their logical quality depends on them. Textbook authors regard enthymematic syllogisms (e.g., "Elvis is a man, so Elvis is mortal") as having an unstated premiss - the major premiss (e.g., "All men are mortal"). They are said to be such because these syllogisms become formally valid when the major premiss is added (i.e., it is a gap-filler). I argue that unstated major premises are not gap-fillers; they support a part of the argument that is already given implicitly - the inference claim (If the premisses are true then the conclusion is true"). As such, their logical status is the same as that of an unstated proposition that supports the minor premiss, which is not part of the argument. Therefore, the so-called "major premiss" is not an unstated premiss of the enthymeme.
\end{abstract}

Informal logic, as a discipline, is distinguished by the attention it gives to everyday, natural, argumentation. Many practioners share the view of Blair and Johnson that people who utter such arguments often fail to state all of the propositions that belong to them:

Natural arguments are usually incomplete. They make leaps from supporting reasons to claims based on them that would be plausible only if certain other assertions, which they do not mention, were also accepted. (Blair \& Johnson 1980:18)

One task of applied informal logic is to reach an accurate judgment of the extent to which an argument provides support for its conclusion. Obviously, this requires the identification of the premisses and conclusion of an argument. We cannot make such a judgment unless we know what the conclusion is, and when we do know it, we cannot judge the quality of the inference if we have not correctly identified all of the premisses:

Anyone who has tried to evaluate natural arguments will know that these missing premisses ... must be formulated, for the strength or weakness of the argument very often depends on what they are. (Blair \& Johnson 1980:18)

The authors recognize that trying to formulate these unstated premisses presents special difficulties:

Anyone who has tried to formulate them or theorized about how to do this will know what a tricky job that can be. Should a missing premiss be trivial, or lend substance to the argument? If the latter, how strong, or weak, should it be? On what grounds is one to answer these questions? The astounding thing is that the intricacies of formulating missing premisses have just not been recognized and addressed. (Blair \& Johnson 1980:18,19)

In the fifteen years since these words were written, there has been some progress made on these issues ${ }^{1}$, but evidently not enough. Recently Mark Weinstein cited "argument recontruction (the problem of missing premisses)" as one of four "vexing theoretic problems" for informal logic (1993:2).

One very common form of everyday argument is the enthymeme. (e.g., "Elvis is a man, so Elvis is mortal"). Traditionally, logicians have conceived of enthymemes as having missing premisses, on the ground that by adding 
an appropriate generalization (e.g., "All men are mortal"), they become deductively valid. These generalizations must be added, it is said, prior to (or in the course of evaluation of an argument. I wish to argue that such generalizations should not be regarded as unstated premisses, since they do not directly support the conclusion. Thus, there is no need to add them for evaluation purposes. So there is a large class of everyday arguments that do not, after all, give rise to a "problem of missing premisses," which means that the problem is less serious than previously thought.

\section{Types of Missing Premisses}

In an article that has become a locus classicus in discussions of the nature and role of missing premisses, Robert Ennis distinguishes between two kinds of "assumptions" that may be missing premisses:

Premise-type assumptions are at least of two types: those that provide backing for a proposition already thought to be part of an argument ("back-ups"), and those that join with one or more other premises in giving support to the conclusion ("gap-fillers") (Ennis 1982:62,63).

In a follow-up to Ennis's paper, Govier argues that gap-fillers ought to be called premisses, rather than assumptions, since there are many assumptions that need to be made in order for arguments to work, but not all of these can be regarded as premisses: "Thus, it is better to speak of missing or unstated premisses rather than of missing or unstated assumptions" $(1987: 82,83)$.

Govier's terminological proposal ought to be adopted, for the reason she gives and also because we traditionally call such propositions "premisses." In what follows I will normally use the term 'unstated' rather than 'missing,' as the latter term has been objected to by some scholars. $^{2}$

Using Ennis's analysis we can say that an argument can have two kinds of unstated premisses: (1) back-ups: propositions that can serve as support for a stated premiss; (2) gap-fillers: propositions that, in conjunction with the stated premisses, make the argument deductively valid. ${ }^{3}$

\section{The Gap-Filler Concept in Textbooks}

Logic textbook authors are primarily concerned with identifying gap-fillers rather than back-ups. This is no doubt because the need for back-ups is a matter of judgment by the individual evaluator, since such judgments depend on what the evaluator knows about the argument topic.

Here are some examples of the kind of gap-filler that textbook writers have chosen to concentrate on. It will be seen that there is some uniformity of interest:

"The stone was an emerald, therefore the stone was green" (Schwartz 1980: example 14, p.147). The unstated premiss is said to be "All emeralds are green."

"The extra cost is about 75 cents per week. Therefore, the extra cost is not much money." The unstated premiss is said to be " $\$ 0.75$ per week is not much money" (Hitchcock 1983:73).

"She's a redhead, so she's probably quick-tempered." The unstated premiss is said to be "Most red-headed women are quick-tempered" (Scriven 1976:81,82).

In each of these cases, the unstated premiss is a generalization. Furthermore, in each case, the arguer is referring to the same thing in both stated premiss and conclusion. These features reflect a preoccupation with arguments that can be construed as syllogisms whose major premiss is unstated. These are what Copi (1982:254) identifies as "first-order 
enthymemes." In what follows I will discuss only these kinds of arguments. The emerald example given above can serve as an illustration when needed.

The specific question I want to address, then, is this: when a syllogistic enthymeme is uttered with its minor premiss stated and the major premiss unstated, is the latter an unstated gapfilling premiss of the argument? I will argue that the major premiss, ${ }^{4}$ whether stated or not, is not a gap-filler at all, but has a backup role, and as such is not an unstated premiss. I will do so based upon a model for single-inference arguments to be developed in the next section.

\section{Modeling One-Inference Arguments}

Given an enthymematic syllogism such as "The stone was an emerald, so the stone was green," textbook writers will commonly represent it in diagram form like Model 1, below.
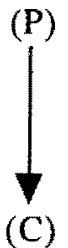

\section{Model I}

This model is intended to represent what is involved in uttering an argument of the form, "P, so C." ('So' can be replaced by any other argument indicator expression that is a logical synonym for 'so'.)

In uttering a one-inference argument, an arguer becomes committed to affirming three claims. Two are obvious: (1) $\mathrm{P}$ is true, and (2) $\mathrm{C}$ is true. While each of these is normally stated explicitly, the third one is always implicit. It can be written in the form, "If
$P$ is true then $C$ is true," or elliptically as, "If $P$ then $C$." This is the inference claim of the argument.

The inference claim is unique as an argument component in that it is, as Govier (1987:96) points out, necessarily implicit. This is so because it always has the form of a conditional having the conjunction of the stated premisses as an antecedent, and the conclusion as a consequent. Suppose we have a simple argument symbolized as "A, so B." We can try to make the inference claim explicit by rewriting the argument as: " $A$; if $A$ then $B$; so $B$." But the inference claim for the rewritten version is "If $A$ and if $A$ then $B$, then B." Thus, when we try to make the implicit inference claim explicit, we produce a new or revised argument having a different implicit inference claim. ${ }^{6}$

While the inference claim must be implicit in the argument as stated, it is nonetheless a component of the argument. The arguer wants the audience to accept the inference claim, plus the stated premisses, so that he/she will accept the conclusion. The arguer's commitment is manifested by the use of some argument indicator word such as 'so', 'therefore', etc.

In Model 1 , the arrow is intended to represent the inference claim. That is, it can be taken to mean, "If P then C." "(P)" means " $P$ is true," and " $(C)$ " means " $C$ is true.' It should not be assumed that each use of the arrow symbol represents an inference claim, however. Consider Model 2:

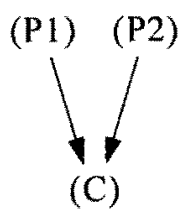

Model 2 
In Model 2, we cannot take the arrows to mean, "If P1 then C" and "If P2 then C." To do so would amount to regarding the diagram as depicting two arguments for C. Model 2 is intended to depict arguments in which each premiss provides some evidence for $\mathrm{C}$, and the conjunction of the two is supposed to be conclusive evidence for $C$. That is, the inference claim is 'If $P 1$ and $P 2$ then $C$.' Therefore, the arrow should be taken to mean, "is evidence/support for.' In the case of Model 1 , since $\mathrm{P}$ is the only premiss, the arrow can be taken to mean, "is conclusive evidence/support for," but this is compatible with stipulating that the arrow means, "is evidence/support for." The argument is simply a special case.

\section{The Role of Major Premisses}

Given the diagramming conventions just set out, how is a syllogism to be diagrammed? Obviously, the minor premiss can be regarded as providing independent evidence/support for the conclusion. In the emerald example, "The stone was an emerald" seems to be good, perhaps conclusive, evidence for, "The stone was green." Thus, we can draw an arrow from " $\mathrm{P}$ " to " $\mathrm{C}$ " in our diagram.

Now, what about the major premiss? Can we simply regard it as "P2" in Model 2? There are both logical and epistemic considerations that suggest that this would be inappropriate.

From an epistemic point of view it can be argued that the major premiss (taken by itself) is irrelevant to $C$, and therefore cannot be evidence/support for it. (This is not the same as being irrelevant to the argument.)

Consider the emerald example. Intuitively, "All emeralds are green." does not appear to be relevant to, "The stone was an emerald." This is probably because the generalization is not explicitly about the stone in question. The evidence given for claiming a thing has a property is usually the fact that it has some other property, or the fact that some causally related state of affairs or event exists/occurs. (Example: evidence for, "The Boston Red Sox home baseball game will be postponed" is, "It is raining in Boston.")

It might be suggested that someone might say, "All emeralds are green, so the stone was green" in a particular context, and the recipient of the argument becomes convinced as a result that the stone was green. Should we say that in such contexts the generalization functioned as evidence? No. In such cases the arguer is relying on the recipient's knowing that the stone was an emerald. In face-to-face contexts, this could be depended upon. But it makes more sense in such cases to say that the recipient consciously (or unconsciously) added the unstated premiss in the course of reaching the conclusion. As genuine evidence for the conclusion in the context, there is no objection to regarding, "The stone was an emerald" as unstated support for it.

I conclude that the minor premiss and the major premiss of a syllogism have different logical roles. ${ }^{7}$ Whereas the minor premiss serves as independent evidence/support for the conclusion, the major premiss does not. Textbook authors are somewhat aware of this difference. Copi, for example, recognizes that, "Not every premiss in an argument provides ... independent support for the conclusion. ... Some premisses must work together to support their conclusion. When this happens, the cooperation they display can be exhibited in the argument's diagram ... by connecting [the premisses] with a brace ... with a single arrow leading from the pair of them to the conclusion" (1982:21).

The problem with this form of depiction is that it does not reflect the different roles that the two premisses have.

If the roles of the two premisses of 
a syllogism differ, and the minor premiss can be seen as evidence/support for the conclusion, what, then, is the role of the major premiss? To identify this we must view the syllogism from the logic viewpoint rather than the epistemic one.

From a logical point of view the form of the "Barbara" syllogism can be written as: "All $S$ is $M$ and all $M$ is $P$, so all $S$ is $P$." The major premiss is the second one. In the emerald example this is, "All emeralds are green." Now, "All $\mathrm{M}$ is P" is logically equivalent to (or at least entails) the conditional "If a thing is $M$ then it is P." This in turn entails, "If this particular thing is $M$ then this particular thing is P." This step represents what is called the rule of "Universal Instantiation" in most versions of the predicate calculus. Thus, in our example about the stone, "All emeralds are green" is logically equivalent to, "If a thing is an emerald then that thing is green." This in turn entails, "If the stone is an emerald then the stone is green." But this last proposition represents the inference claim of the enthymematic version of the argument (the version containing only the minor premiss as a premiss).

Thus, the major premiss entails the inference claim of the enthymeme. That is to say, it provides (if true) conclusive evidence/support for that inference claim. And this being so, it cannot be conclusive evidence/support for the conclusion itself, since "If $P$ then $C$ " is not logically equivalent to $C$, nor does it entail $C$.

We now have the answer as to how to accomodate "Major Premisses" in the adopted diagram format. They cannot be represented as "P2" in Model 2, and since they support the inference claim of the enthymeme, which is represented by the arrow from $P$ to $C$, it is appropriate to draw an arrow from the major premiss, (MP), to the arrow between $P$ and $C$, as shown in Model 3, below.

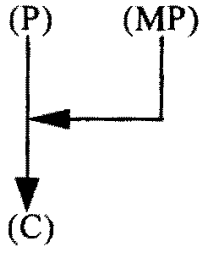

\section{Model 3}

For this paper, the most important point to be derived from the exercise of establishing how to diagram a syllogism is that, when the major premiss is unstated (i.e., when we have an enthymeme) and we choose to call it an unstated premiss of the argument, its role in the argument is not that of a gap-filler, but as a back-up. According to Ennis, it will be recalled, a backup "... provides backing for a proposition already thought to be part of the argument..." (Ennis 1982:62). That proposition is, in this case, the implicit inference claim.

Can an enthymeme be a complete argument by conceptual standards? That is, given our concept of an argument, is an enthymeme such as "The stone was an emerald, so the stone was green" a clear instance of an uttered argument, or is it a borderline case, or even a non-case by virtue of missing a part?

Textbook authors generally define 'argument' in such a way that enthymemes do count as clear instances. Copi's definition is typical of those found in logic textbooks: "An argument... is any group of propositions of which one is claimed to follow from the others, which are regarded as providing support for or grounds for the truth of that one .... The simplest kind of argument consists of just one premiss and a conclusion ..." $(1982: 6,7)$.

Textbook authors, then, seem prepared to accept arguments with one premiss as completely-stated. And just as we can have a complete argument without any backing for the single premiss, by parity of reasoning it would seem that no 
backup is required for the inference claim either. Thus, an enthymematic syllogism is a complete argument. (Of course it is not a complete syllogism.)

In referring to completeness here I am talking about conceptual completeness. However, the case for major premisses as needed parts of an enthymematic argument involves the notion of logical completeness, not conceptual completeness. I will evaluate those arguments shortly, but first I want to mention some pragmatic considerations in favor of not regarding major premisses as unstated premisses of enthymemes.

If major premisses have the role shown in Model 3, we can explain why people frequently utter enthymemes rather than complete syllogisms, and why, as Hitchcock observes, "we are unaware of having omitted a premiss when we advance an enthymeme" (Hitchcock 1985:94). That is, they are a backup for the argument that only get stated when the validity of the inference is challenged.

Arguments are frequently uttered with a view to persuading someone else to accept a claim that we judge they believe is dubious or false. In the interests of logical efficiency we utter only the propositions that we think are necessary to persuade. If we think the audience will accept a premiss as true because of information they have that supports it, we do not provide any backup for the premiss. Furthermore, if we think they might regard that premiss as providing adequate support for the conclusion (i.e., we think they would regard the inference claim as true), we do not state the major premiss as another premiss. It, and premiss backups, are considered to be logically redundant in the context.

Our argumentation practice of stating arguments enthymematically, then, is explained by an intuitive understanding that major premisses are backups for inference claims, and when we fail to state the gap-filler we are manifesting our belief that the audience will accept our inference claim, that is, that they will regard $\mathrm{C}$ as following from $\mathrm{P}$ because of information they already have. Were I to try to prove to a relative that a missing heirloom ring contained a green stone, and I know that this person is knowledgeable about gems, I might give as a reason for this claim, "The stone was an emerald." I would see it as unnecessary to also state the backup "All emeralds are green," if I believe my relative knows this.

\section{The Case for the Gap-Filler Interpretation}

Before detailing some objections, conceptual and methodological, to the gapfiller approach to argument evaluation, it is appropriate to consider the grounds given in support of saying that major premisses are unstated premisses of syllogistic enthymemes.

The main one is contained in the first quote from Blair and Johnson: gap-fillers are relied on to infer $C$ from $P$.

This seems to be true, but it must be remembered that to prove their conclusions, arguments rely on many propositions' being true, most of which are clearly not premisses. ${ }^{8}$ In any case, if major premisses are really backups for inference claims, the question of whether they are unstated premisses is the same one that arises for backups for stated premisses. Are backups for stated premisses to be regarded as unstated premisses?

In terms of their logical properties, we have no way to distinguish backups that are premisses from ones that are not. If we take every backup to have its own backup when added to the argument, we must take the view that any argument has an indefinite number of premisses. If instead we deny that there is an infinite regress, then we must take some such view 
as that a completely stated argument must begin with self-evident premisses. The former choice is absurd and the latter is not much better. It is better to adopt a third choice: we take the argument to be complete with the original stated premisses. And since the major premiss is really a backup for the inference claim, we are led to the view that an uttered argument can be complete without it.

It will be objected here that major premisses, even if it be granted that they are backups, are a special kind of backup. That is, their addition to an argument produces an argument with a valid inference. And since an argument must have a valid inference if it is to prove its conclusion, the gap-filler is an unstated premiss in any argument that is not formally valid as it stands.

This thesis is questionable. Another way of stating it is to say that an inference claim is only acceptable when it is necessarily true by virtue of its logical form. But an argument must be judged as proving its conclusion whenever we accept its stated premisses and its inference claim as true, whether necessarily or contingently. Perhaps some examples of arguments with these kinds (or degrees) of validity will clarify this claim.

An example of an argument that is formally valid: "The stone was an emerald, and all emeralds are green, so the stone was green." It is formally valid because it can be written in such a way as to correspond to a valid syllogism pattern, and to a valid pattern in first-order predicate calculus.

However, arguments can be deductively valid without being formally valid. Consider an example of an argument that has a necessarily true inference claim, but truth arises from the meaning of the words used to state it: "Al is older than Bill, Bill is older than Charlie, therefore, Al is older than Charlie." Copi says that the proposition, "Being older than" is a transitive relation" is a missing premiss because it is needed to make the argument formally valid (1972:155). Trudy Govier challenges this interpretation:

But this must be wrong. The argument $\ldots$ is in fact deductively valid as stated. Copi's account goes beyond deductivism in that he seeks to make the argument not just deductively valid, but deductively valid in virtue of its form as identified within a specific system of logic. The stated premisses do entail the conclusion. It is just not formally provable that this is the case. (Govier 1987:93)

Besides cases in which we have deductive validity without formal validity (following Govier, we can call these "semantically" valid), there are those in whch the inference claim is contingently true. For example: "The stone was an emerald, so the stone was green." The backing for the inference claim is "All emeralds are green," which is true, so the inference claim is true. However, in some logically possible worlds the generalization is false, so that the truth of the premiss does not guarantee the truth of the conclusion in those worlds. The argument is valid only in those worlds in which all emeralds are green. For these reasons, such examples can be called "contingently" valid (or, following Govier, "materially" valid).

Thus, the gap-filler method involves the use of a standard (formal validity) beyond that required for proof. The use of such a standard results in the search for unstated premisses in cases in which there are none required, specifically, when semantic rules or empirical facts ensure that the inference claim is true. This must be regarded as a serious defect in the approach.

Another problem with the gap-filler method is that adopting it commits us to several unpalatable positions: (1) all fully-stated arguments are formally valid, (2) argument evaluation is solely a matter of premiss evaluation, (3) there are no fallacies, only arguments with unstated premisses. The first seems to be 
incompatible with much of what has been said about the nature of logic as a subject, i.e., that applied logic is establishing whether or not inferences are valid. The second position follows from the first, and given the role of applied logic as just described, it would follow that argument evaluation is not the main business of logic! The third position also follows from the first, and is incompatible with the theory and practice of those engaged in the study of fallacies. (See Govier 1987:85.)

A methodological difficulty in using the method is in the formulation of the gap-filler. The proposition identified as the gap-filler will frequently be a stronger claim than we may want to impute to the arguer, given the information we think the arguer has. For example, if an argument is a syllogistic enthymeme of the "Barbara" type, the gap-filling major premiss will be a universal generalization. Suppose we are in a world in which about $1 \%$ of the emeralds in circulation are blue and the rest are green, and this is general knowledge. Given the argument, "The stone was an emerald, so the stone was green," the proponents of the gap-filler approach would identify "All emeralds are green" as the gap-filler that secures formal validity. The arguer who knows of the existence of the blue emeralds would not find this claim acceptable, and would deny that it is a premiss in his or her argument.

Someone who supports the gap-filler approach would respond that the arguer must accept the generalization as a premiss, otherwise his/her argument does not have a valid inference. This is correct. It is neither formally valid, nor semantically valid, nor contingently/materially valid. But the arguer could respond that the stated premiss, although it does not entail the conclusion, is a very good reason to believe the conclusion.

At this point a deductivist might complain that there is an unstated qualifier in the conclusion. That is, the arguer ought to have said something like: "The stone was an emerald, so, almost certainly, the stone was green." The addition of the qualifier can be seen as justified by the truth of the strongest defensible inference backup: "Nearly all emeralds are green."

While the deductivist seems to be right about imputing unstated qualifiers in cases like this, qualifiers are not premisses. We can regard the major premiss as entailing the inference claim of the argument with its unstated qualifying expression added, but the major premiss itself is still backing for the inference claim and as such is not a gapfiller.

\section{An Alternative Approach}

This "unstated qualifier" approach to argument evaluation points to a more adequate method of evaluating inductive arguments when the arguer is not present to elaborate on her/his argument. When there are no qualifiers present, we follow this procedure for syllogistic enthymemes in which the stated premiss and the conclusion have the same referent:

(1) Write the backup for the inference claim in hypothetical form, e.g., "If a stone is an emerald then that stone is green." This is done by simply making the referent of the antecedent and consequent denote the class to which the referent of the stated premiss and conclusion belong. We make the predicate of the antecedent the same as the predicate of the stated premiss, and the predicate of the consequent the same as that of the conclusion.

(2) Assign a probability value to the backup, noting that $p($ If $X$ then $Y)=p(Y /$ $X)$. For instance, in the example we need a value for: $p$ (a stone is green/the stone is an emerald). In the world in which $1 \%$ of emeralds in circulation are blue, and 
there are no considerations that favor one shade over the other in this context, the value is 0.99 .

(3) Since the backup entails the inference claim, $p($ If $P$ then $C)(=p(C / P))$ equals the probability of the backup. For the example, $\mathrm{p}(\mathrm{C} / \mathrm{P})=0.99$.

(4) The product " $\mathrm{p}(\mathrm{P}) \times \mathrm{p}(\mathrm{C} / \mathrm{P})$ " represents the probability of the conclusion, $\mathrm{p}(\mathrm{C})$ ), given the argument, or to put it differently, the "degree of support" provided by the argument. For the example, if we suppose that $\mathrm{P}$ is true, $\mathrm{p}(\mathrm{C})=1 \times 0.99=$ 0.99 . This outcome correctly reflects the judgment that the premiss is very good evidence for the conclusion.

This probabilistic approach is highly appropriate for inductive argument evaluation. It incorporates a role for the socalled "gap-filler" as a backup to the inference claim, although not the role envisioned by those who advocate regarding it as an unstated premiss. However, the approach just outlined is not subject to the criticisms raised against the gapfiller approach, and it has virtues of its own.

In particular, it involves genuine inference evaluation. We take an enthymeme as a complete argument as stated, so that issues about reconstruction do not arise. To the extent that these issues are identical with "the problem of unstated premisses," as Weinstein thinks, one of the main stumbling blocks to implementation of the informal logic program is removed when we understand the correct role of major premisses.

\section{Conclusion}

I have argued that the major premisses of syllogisms do not function as unstated "gap-fillers" for the corresponding enthymemes, contrary to the views expressed in some popular logic textbooks. I have argued that their role is not that of gap-filling, but one of backing up inference claims. One consequence of this view is that they are not unstated premisses of their enthymemes. This would be true of any proposition that qualifies as the inference claim backup of any argument, syllogistic or otherwise. They may, however, have a role in the evaluation of the argument, as sketched in the last section.

I want to make it clear that my argumentation does not (and could not) show that arguments never have unstated premisses. A clear counter-example is an argument in which only conclusion and warrant are stated, e.g., "All emeralds are green, so the stone was green." In my view, to represent a complete argument, an utterance must include both a conclusion and a reason to believe it. In such a case we will be correct in saying that, "The stone was an emerald" is an unstated premiss.

To end on an historical note: in the history of syllogistic there has been a tendency to overlook the asymmetry between the premisses in their relation to the conclusion. This is probably a legacy of Aristotle, who used the same term (protasis) to refer to both. This has led to a tension, if not an inconsistency, in most "Copi-style" introductory logic texts. Copi himself, for instance, defines 'premisses' as "propositions which are affirmed as providing support or reasons for accepting the conclusions" (1982:7), Subsequently he adopts this (partial) definition of 'syllogism': "A syllogism is a deductive argument in which a conclusion is inferred from two premisses" (1982:210, my stress). Later, however, in his presentation of the predicate calculus he introduces the rule of "Universal Instantiation" (1982:367). According to this rule, given (for example), "All emeralds are green" as a major premiss, we can infer "If this is an emerald then this is green." Thus, in effect, a major premis s considered by itself is regarded as 
conclusive evidence (if true) for a conditional containing the conclusion, not for the conclusion per se.

As these quotes from Copi show, some of the confusion about unstated/missing premisses is due to the use of the term 'premiss.' This being so, it is a pity that Toulmin's work on the distinction between minor and major premiss has not gained greater currency. ${ }^{10}$

\section{Notes}

1 For articles on the subject see the bibliography by Hansen (1990). He explicitly identifies the items that are on the topic.

2 Gough and Tindale (1983), for example, argue that these premisses are "hidden," not missing, but they would agree that they are unstated.

Note that, as Ennis recognizes, this is a criterion for gap-fillers, not a definition. He presents three "criteria for needed assumptions": fidelity, contributing to gap-filling, and plausibility $(1982: 70,71)$. The criterion I have singled out is the second one, which is clearly a necessary condition for a proposition to qualify as a gap-filler. Given the complexity of the criteria, we should not regard the major premiss as the only candidate for gap-fillers for enthymemes. As a referee for Informal Logic points out, the enthymeme, "This stone is an emerald, so this stone is green" can be made deductively valid by adding, for example, "It is not the case that this stone is an emerald which is not green."

My interest here is not whether there are other candidates that meet the criterion expressed here, but whether the major premiss is itself an unstated premiss, given that it meets the criterion.

It may be worth noting here that the textbook writers whose examples are discussed in this paper, and most others, probably regard the major premisses of syllogisms to be paradigm cases of gap-fillers for their corresponding enthymemes. As such, any correct definition of 'gap-filler' can be tested by reference to them.

4 I will conform to standard usage and use the expression 'major premiss' throughout, even though I will argue that they are not

\section{premisses of the corresponding enthymemes.}

5 There is controversy in some quarters about how "If ..., then ..." is to be interpreted. To represent the inference claim, it needs to be taken in the sense "The truth of ... guarantees the truth of ... ," where the guarantee arises from a causal law (e.g., "If it has been below freezing all night then the puddles have ice on them"), a conceptual/semantic connection (e.g. "If today is Tuesday then tomorrow is Wednesday"), or some other factor re sponsible for there being a connection between antecedent and consequent. "Guarantee' is a good term of interpretation here because it implies that the consequent becomes true when the antecedent becomes true. The material implication interpretation of "If ... then ..." is inadequate precisely because it fails to capture the notion of connection. It is not a great exaggeration to claim that it is seen as a plausible interpretation only by those who wish to show that formal logic systems can be used in the evaluation of arguments in natural languages.

- This feature of arguments was brought to the attention of logicians by Lewis Carrol (Carrol 1896).

7 It may have been these epistemic considerations that led Stephen Toulmin to recognize that the minor premiss and major premiss of syllogisms have different roles. In providing an analysis of argument structure, which he applied specifically to the syllogism, Toulmin labeled the conclusion the "claim" and the minor premiss the "data" (later, he changed this to "ground"). The major premiss could be interpreted as either a "warrant" or "backing" for the war- 
rant. He makes it clear that the backing for a warrant has the same epistemic status or logical form as the datum/ground: "... the backing for warrants can be expressed in the form of categorical statements of fact quite as well as can the data appealed to in direct support of our conclusions" (1958:105). However, he is not quite so clear in describing the logical form of warrants. Sometimes generalizations are said to be warrants (e.g., "A man born in Bermuda will generally be a British subject" (1958:102)). Sometimes he calls indicative conditionals warrants. (e.g., "If anything is red, it will not be black." (1958:98)) More frequently, though, he uses rule-stating language, and at one point he refers to warrants as "... propositions of a different kind: rules, principles, inference-licenses ..." (1958:98). Thus, the major premiss form, "All A's are B" can be interpreted as, "... every single $A$ has been found to be a $B$..., "i.e., as backing, or as, "... an A can be taken certainly... to be a B ... " a warrant (1958:111).

It will become clear that I do not distinguish the minor premiss from the major premiss in the way Toulmin does. In particular, I do not give the major premiss the warrant interpretation, that is, I do not treat it as a rule, or formulate it as such. Rather, I operate with the traditional view that it is a propsition providing epistemic/logical sup. port for some component of the syllogism, since this is the view of those whose interpretation of major premisses I am challenging.

8 For a comprehensive list of kinds see Govier (1987:92).
\& There is said to be a problem in specifying gap-fillers in such a way as to uniquely determine them. Ennis tries to deal with this by presenting his three criteria for needed premisses. It is tempting to specify that the inference claim backup must be the most defensible claim that provides adequate support for the inference claim, as this promotes charity in the identification of unstated premisses. This principle could be interpreted logically as a requirement to take the backup to be the logically "closest" proposition that entails the inference claim, and that the arguer will accept. By logically "closest" I mean the generalization that has the smallest scope. This will frequently be the major premiss for an enthymematic syllogism.

One suggested candidate is: "It is not the case that both $\mathrm{P}$ and not C." Although it is the weakest claim that may entail the inference claim. it is not epistemically adequate. What is wanted is a proposition that is more plausible than the inference claim, but if it is taken as logically equivalent to the inference claim (not my view) it will not be more plausible because it will have the same truth conditions. If we do not take them as logically equivalent, on the ground that the negated conjunction does not capture all of the "content" of the conditional, the former does not entail the latter, so it cannot serve as backup.

10 I wish to thank David Hitchcock, one of the referees of this paper for Informal Logic, for his incisive and detailed comments on the originally-submitted version of this paper.

\section{Bibliography}

Blair, A., \& Johnson, R., eds. (1980) Informal Logic: The First International Symposium. Inverness, CA: Edgepress.

Carrol, L. (1896) "What the Tortoise Said to Achilles". Mind 4, 1895.

Copi, I. (1972) Symbolic Logic (3rd ed). New York: MacMillan.

Copi, I. (1982) Introduction to Logic (6th ed). New York: MacMillan.
Ennis, R. (1982) "Identifying Implicit Assumptions". Synthese 51, 61-86.

Gough, J. \& Tindale, C. (1985) "Hidden' or 'Missing' Premises". Informal Logic 7 (2\&3), 99-107.

Govier, T. (1987) Problems in Argument Analysis and Evaluation. Dordrecht-Holland: Foris.

Hansen, H.V. (1990) "An Informal Logic Bibliography". Informal Logic 12(3), 155-184. 
Hitchcock, D. (1983) Critical Thinking: A Guide to Evaluating Information. Toronto: Methuen.

Hitchcock, D. (1985) "Enthymematic Arguments". Informal Logic 7(2\&3), 84-97.

Schwartz, T. (1980) The Art of Logical Reasoning. New York: Random House.

Scriven, M. (1976) Reasoning. New York: McGraw-Hill.

Toulmin, S. (1958) The Uses of Argument. Cambridge: Cambridge University Press.
Weinstein, M. (1993) "Critical Thinking and One-Night Stands: A Reply to Scriven". $C T$ News 11(2), 1-6.

WAYNE GRENNAN

DEPARTMENT OF PHILOSOPHY

SAINT MARY'S UNIVERSITY

HALIFAX, NOVA SCOTIA

$B 3 H 3 C 3$ 